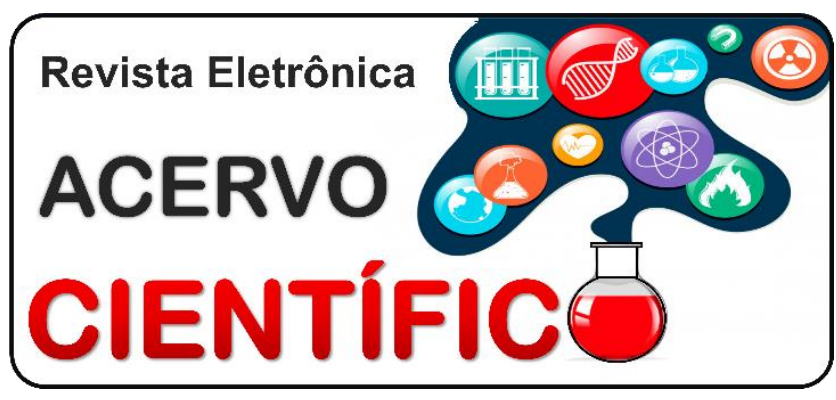

\section{REVISÃO BIBLIOGRÁFICA}

Recebido em: 10/2020

Aceito em: $12 / 2020$

Publicado em: 1/2021

\title{
Cesárea e depressão pós-parto: uma revisão dos fatores de risco
}

\author{
Cesarean section and postpartum depression: a risk factors' review
}

Cesárea y depresión posparto: una revisión de los factores de riesgo

\begin{abstract}
Mariana Olympio Rua ${ }^{*}$, Gustavo Fonseca Genelhu Soares², Caroline Silva de Araujo Lima ${ }^{3}$, Eduardo Rodarte Martins ${ }^{4}$, Leonardo Fávaro Pereira ${ }^{5}$, Natália Tiemi Nakahata ${ }^{6}$, Lívia Pêpe Leão da Rocha $^{7}$, André Felipe Costa Alves ${ }^{8}$, João Pedro Kim Amamura ${ }^{9}$, Júlia Carneiro Leão ${ }^{10}$.
\end{abstract}

\begin{abstract}
Resumo: Este artigo buscou analisar, por meio de revisão de literatura, a inter-relação entre parto cesariano e depressão pós-parto, avaliando causas, fatores de risco e reflexos. A depressão pós-parto é um distúrbio mental que afeta entre 10 e $20 \%$ da mulher e é observada, sobretudo, em gestante que experimentaram sintomas relacionados ao estresse, à ansiedade e à depressão, podendo também estar associado a condições sociais. A cesárea está associada a alterações biológicas no corpo da mãe, como variação do nível sérico de hormônios, de prolactina e de interleucina-6, a hemorragia, além de sensações de medo e dor. Mulheres primíparas, sobretudo com mais de 30 anos de idade, correm maior risco de apresentarem depressão pós-parto, assim como aquelas que tiveram uma gravidez indesejada ou não intencional. Além das repercussões do quadro depressivo para a mulher, o que inclui ideações suicidas e alterações na função sexual, há maior risco de infanticídio, atraso no desenvolvimento de habilidades de comunicação do bebê e aumento nos gastos com saúde. Assim, verificou-se a necessidade de a depressão pós-parto ser considerada por clínicos, visto que pode surgir antes ou depois do parto, ressaltando a importância da avaliação precoce, a qual pode incluir intervenções psicológicas, farmacológicas e hormonais.
\end{abstract}

Palavras-chave: Depressão pós-parto, Cesárea, Transtornos mentais.

Abstract: This article aims to analyze, through a literature review, the interrelationship between cesarean section and postpartum depression, assessing causes, risk factors and reflexes. Postpartum depression is a mental disorder that affects between 10 and $20 \%$ of women and is observed, especially, in pregnant women who have experienced symptoms related to stress, anxiety and depression, and may also be associated with

\footnotetext{
1 Universidade Vila Velha (UVV), Vila Velha - ES. *E-mail: marilympio@gmail.com

${ }^{2}$ Centro Universitário de Caratinga, Caratinga - MG.

${ }^{3}$ Faculdade Dinâmica Vale do Piranga (FADIP), Ponte Nova - MG.

4 Faculdades Integradas do Norte de Minas (FUNORTE) - Montes Claros - MG.

${ }^{5}$ Universidade Federal do Espírito Santo (UFES), Vitória - ES.

${ }^{6}$ Centro Universitário Ingá (UNINGÁ), Maringá - PR.

7 Instituto Metropolitano de Ensino Superior do Vale do Aço (UNIVAÇO), Ipatinga - MG.

${ }^{8}$ Centro Universitário de Brasília (UNICEUB), Brasília - DF.

9 Universidade Cidade de São Paulo (UNICID), São Paulo - SP.

${ }^{10}$ Centro Universitário de Volta Redonda (UNIFOA), Volta Redonda - RJ.
} 
social conditions. Cesarean section is associated with biological changes in the mother's organism, such as variation in the serum level of hormones, prolactin and interleukin-6, hemorrhage, in addition to feelings of fear and pain. Primiparous women, especially over 30 years old, are at greater risk of developing postpartum depression, as well as those who have had an unwanted or unintended pregnancy. In addition to the repercussions of depressive conditions for women, which include suicidal ideations and changes in sexual function, there is a greater risk of infanticide, delay in the development of the baby's communication skills and increased health costs. Thus, there was a need for postpartum depression to be considered by clinicians, since it may appear before or after delivery, emphasizing the importance of early assessment, which may include psychological, pharmacological and hormonal interventions.

Keywords: Depression, postpartum, Cesarean section, Mental disorders.

Resumen: Este artículo buscó analizar, a través de una revisión de la literatura, la interrelación entre el parto por cesárea y la depresión posparto, evaluando causas, factores de riesgo y reflejos. La depresión posparto es un trastorno mental que afecta entre un 10 y un $20 \%$ de las mujeres y se observa, sobre todo, en mujeres embarazadas que han experimentado síntomas relacionados con estrés, ansiedad y depresión y estar también con condiciones sociales. La cesárea se asocia con cambios biológicos en el cuerpo de la madre, como variación en el nivel sérico de hormonas, prolactina y interleucina-6, hemorragia, y sentimientos de miedo y dolor. Las mujeres primíparas, especialmente las mayores de 30 años, tienen mayor riesgo de desarrollar depresión posparto, así como aquellas que han tenido un embarazo no deseado. Además de las repercusiones de las condiciones depresivas para la mujer, que incluyen ideas suicidas y cambios en la función sexual, existe un mayor riesgo de infanticidio, retraso en el desarrollo de las habilidades comunicativas del bebé y mayores costos de salud. Por lo tanto, era necesario que los médicos consideraran la depresión posparto, enfatizando la importancia de una evaluación temprana, que puede incluir intervenciones psicológicas, farmacológicas y hormonales.

Palabras clave: Depresión posparto, Cesárea, Trastornos mentales.

\section{INTRODUÇÃO}

É inegável a importância obstétrica da cesárea, procedimento que já salvou milhares de mães e recémnascidos. Nesta perspectiva, com os avanços tecnológicos e sociais, a cesárea passou de uma operação obstétrica específica para emergências para um modo relativamente comum de concepção humana (CHEN $\mathrm{HH}$, et al., 2017; MOAMERI H, et al., 2019). As taxas de realização desse procedimento em todo o mundo são crescentes, excedendo em muitos locais $20 \%$ do total de partos, taxa significativamente maior do que a taxa de $15 \%$ considerada a mais alta aceitável pela Organização Mundial da Saúde (OMS) (CHANG SR, et al., 2015). Os números crescentes podem ser associados ao número cada vez maior de mulheres que solicitam uma cesariana eletiva, muitas vezes decorrente do medo do parto vaginal, sensação experimentada por aproximadamente $20 \%$ de todas as mulheres grávidas (OLIEMAN RM, et al., 2017).

Entretanto, a realização desta sem indicação médica pode acarretar complicações para a mãe e para o recém-nascido, tais quais problemas no sistema imune do recém-nascido (RN), redução da fertilidade materna, aumento das chances de aborto espontâneo e, por fim, aumento da chance de quadros de ansiedade e depressão pós-parto (DPP) (CHANG SR, et al., 2015; MOAMERI H, et al., 2019). A DPP é um distúrbio do humor com efeitos agudos ou crônicos e que tem impacto não apenas sobre a saúde da mulher, mas também sobre suas famílias e sobre a sociedade na qual está inserida (SADAT Z, et al., 2014). Esse transtorno afeta $10-20 \%$ das mulheres, podendo iniciar a qualquer momento durante o primeiro ano após o parto e durar vários meses. O quadro clínico inclui sintomas de mudança de sono e na alimentação, tristeza, ansiedade, redução da libido, fadiga, sentimentos de solidão e labilidade emocional (ILSKA M, et al., 2020).

A DPP se desenvolve a partir de um eixo multifatorial que inclui histórico de depressão pré natal, má relação matrimonial e falta de apoio social, além de questões como baixa renda e eventos familiares marcantes (CLOUT D e BROWN R, 2015; CIRIK DA, et al., 2016). Geralmente se expressa quando a mãe 
está em casa e longe dos cuidados especializados - as novas mães muitas vezes não reconhecem as mudanças psicológicas que sofrem por acreditarem que devem estar necessariamente felizes e que não devem expressar preocupações (ATAN ŞU, et al., 2018).

É importante destacar, porém, que o termo "depressão pós-parto" perdeu sua especificidade, sendo usado para determinar qualquer caso de depressão no período após o parto, independentemente dos sintomas apresentados - os sintomas da DPP diferem dos da "depressão clássica", tais quais probabilidade elevada de ansiedade concomitante, sintomas obsessivos-compulsivos, menor taxa de suicídio, má resposta ao tratamento e necessidade de farmacoterapia (ZACONETA AM, et al., 2013).

O presente estudo objetiva avaliar os fatores de risco que contribuem para a depressão pós-parto, além de avaliar sua relação com o parto cesariana.

\section{REVISÃO BIBLIOGRÁFICA}

\section{A Depressão Pós-Parto}

Várias mudanças drásticas ocorrem desde o início gravidez até o pós-parto, incluindo mudanças biológicas, psicológicas e comportamentais. A depressão é uma complicação comum durante a gravidez e o período pós-parto. Mais de $70 \%$ das mulheres expressam sintomas de depressão durante a gravidez e 10$15 \%$ das mulheres relatam sintomas de DPP (TATA N, et al., 2019). No Brasil, a prevalência do transtorno varia de 7,2 a $43 \%$ (ZACONETA AM, et al., 2013).

Os sintomas de DPP podem ocorrer a qualquer momento no período pré e pós-parto. Estes incluem tristeza, ansiedade, baixa autoestima, culpa, vazio, retraimento social, pouca energia, sensação de estar sobrecarregado, sono e distúrbios alimentares, sensação de inadequação para cuidar do bebê e pensamentos obsessivos, além de efeitos negativos na relação mãe-bebê, nutrição inadequada e crescimento insatisfatório do recém-nascido. Ademais, as mães que apresentam DPP são mais suscetíveis à depressão em períodos tardios de suas vidas (QUAN C, et al., 2019; MAHISHADE AV e BHATT JA, 2017; SADAT Z. et al.,2014; ZACONETA AM, et al.,2013).

Um artigo publicado em Taiwan constatou sintomas de estresse em 7,3\% das mulheres, ansiedade em $19 \%$, depressão em $5,9 \%$ e pelo menos um dos transtornos citados em $27,6 \%$ nos pacientes submetidos à cesárea. Em mulheres que tiveram parto normal as taxas de estresse $(5,3 \%)$, ansiedade $(16,6 \%)$ e depressão $(4,5 \%)$ foram mais baixas, se comparadas com mulheres que tiveram cesárea ( $C H E N ~ H$, et al., 2017; ZACONETA AM, et al., 2013).

De acordo com uma meta-análise, as mães que se submetem ao parto normal têm menor risco de DPP do que as mães com cesárea e o risco de DPP após a cesárea eletiva é maior do que o risco após o parto normal e a cesárea de emergência. Um estudo chinês verificou que mulheres com algumas tendências de transtornos mentais podem escolher a cesárea com mais frequência. Assim, não a cesárea em si, mas o histórico fisiológico que leva à cesárea também pode estar associado à depressão em algumas mulheres (QUAN C, et al., 2019).

Sem dúvida, o medo do parto é o principal fator que leva à cesárea eletiva. Cerca de $80 \%$ das mulheres grávidas podem experimentar medo do parto e 13\% delas experimentam medo do parto grave. Os fatores negativos associados ao medo do parto são distúrbios do sono, depressão pré-natal, aumento do uso de assistência pré-natal e solicitação materna de cesariana eletiva (TATA N, et al.,2019). Um estudo prospectivo realizado em gestantes internadas para atendimento pré-natal em centros de saúde revelou que mais da metade $(56,2 \%)$ das mulheres com medo do parto não tinha ansiedade ou depressão, mas ansiedade e depressão aumentaram a prevalência de medo do parto e indiretamente, o percentual de cesarianas (QUAN C, et al.,2019; TATA N, et al.,2019).

Um estudo transversal do Irã descobriu que o DDP foi maior entre as mães submetidas a cesárea $(21,17 \%)$ em comparação com as mães submetidas parto vaginal (8,23\%). Dentre as mulheres que realizaram cesárea, $10,5 \%$ apresentaram sintomas depressivos por desejarem o parto natural e dor no local da sutura, 5,88\% 
devido à dor no local da sutura e $4,70 \%$ devido à dor no local da sutura e problema de alimentação. Das mães com parto normal que tiveram depressão, 4,70\% apresentaram características depressivas devido à dor da episiotomia e distúrbios do sono (MAHISHADE AV e BHATT JA, 2018; ZACONETA AM, et al., 2013).

É inegável que o declínio abrupto no nível sérico de hormônios que segue a expulsão da placenta está associado a mudanças de humor e sintomas depressivos e por consequência, as puérperas são mais susceptíveis a mudanças de humor (ZACONETA AM, et al., 2013). Uma pesquisa iraniana constatou que $24 \%$ das mães entrevistadas que realizaram parto vaginal tiveram sintomas depressivos na primeira avaliação, em contraste a $20,6 \%$ das submetidas à cesárea. Após dois meses, em uma segunda análise, $12,6 \%$ das mães que tiveram parto vaginal apresentaram ansiedade, enquanto na cesárea $16,6 \%$ das mulheres tinham sinais de DPP (SADAT Z. et al.,2014).

Em um estudo Brasileiro, de 107 mulheres entrevistadas, 5,6\% apresentavam sintomas depressivos antes e depois do parto, 4,7\% apresentaram sintomas apenas antes do parto e 5,6\% apenas após o parto, sendo as mães solteiras mais susceptíveis (ZACONETA AM, et al.,2013).

\section{Transtornos mentais relacionados à cesárea}

Em um estudo retrospectivo, do qual 38.000 gestantes participaram, foram identificados 16.639 casos com pelo menos um diagnóstico de transtorno mental: $16,9 \%$ de transtorno de ansiedade, $24,2 \%$ de transtorno somatoforme/dissociativo e $11,7 \%$ de reações agudas de estresse. No mesmo estudo, mulheres com diagnóstico de transtorno mental eram mais propensas a ter seus filhos por cesariana. Além disso, bebês de mulheres diagnosticadas com depressão durante a gravidez eram mais propensos a ter baixo peso e / ou ter parto prematuro (WALLWIENER S, et al., 2019).

Outro estudo de coorte, conduzido em Taiwan, evidenciou que a incidência para pelo menos uma das três doenças é de $2,76 \%$ na cesariana, e de $2,34 \%$ no parto vaginal, o que é considerado uma diferença estatisticamente significativa. Com isso, fica claro que o estresse, a depressão e a ansiedade são os transtornos mentais funcionais mais comuns após o parto. O mesmo artigo relacionou possíveis causas para DPP a falta de apoio social e depressão pré-natal, baixa escolaridade, desemprego e problemas crônicos de saúde (CHEN HH, et al., 2017).

Ademais, há relações entre a ocorrência de ansiedade e/ou depressão pós-parto com características demográficas: mulheres que fumaram exclusivamente durante a gravidez tiveram a maior prevalência de ansiedade pós-parto $(21,6 \%)$, e de ansiedade e depressão pós-parto simultaneamente $(21,4 \%)$, gestantes que tomaram 7 ou mais bebidas alcoólicas por dia antes da gravidez tiveram maior prevalência de depressão pós-parto (7,5\%) (FARR SL, et al., 2014). A própria cesariana, a decepção com o tipo de parto e um período de recuperação mais prolongado podem também predispor à depressão pós-parto, por meio do aumento do estresse, queixas somáticas e baixa autoestima (c). Tendo em vista a alta prevalência de desordens mentais em gestantes, é imensurável a necessidade de políticas de saúde que provam a qualidade do trabalho de parto e o parto em si para que se obtenha efeitos positivos para a mãe e o bebê, e também ao desenvolvimento sadio do bebê (BELL AF, et al., 2016).

\section{Fatores de risco para Depressão Pós-Parto}

Durante a gravidez, parto e puerpério, as mulheres passam por mudanças físicas e psicológicas adaptativas, que os tornam vulneráveis a distúrbios psicológicos. Além das mudanças no sistema reprodutivo e uma reversão para fisiologia não grávida, ocorrem mudanças psicossociais após o parto, a transição para a maternidade, o restabelecimento de relacionamentos e trabalho para atender às necessidades físicas $e$ emocionais de um recém-nascido e outros membros da família. Se não mitigados, os estressores pós-parto podem levar à fadiga, ansiedade e diminuição do autocuidado; esses fatores foram associados a um risco aumentado de doença física e mental, incluindo a DPP (ZANARDO V, et al., 2017).

Vários fatores podem predizer diferentes sintomas depressivos e de ansiedade em mulheres que realizam o parto. Entre eles, fatores como peso corporal e qualidade do sono são de particular importância clínica. Mulheres obesas tendem a apresentar níveis mais elevados de sintomas depressivos e de ansiedade após o 
parto. Dificuldades para dormir durante o final da gravidez estão associadas a humor e ansiedade no período perinatal. A maioria das pesquisas até agora focou principalmente em correlatos de sintomas depressivos ou de ansiedade, preditores de trajetórias de sintomas depressivos e de ansiedade (KUO S, et al., 2014).

Relatos de depressão e ansiedade foram comparados, e mostraram que a primiparidade estava relacionada a altos níveis de ansiedade-estado, sugerindo que a primiparidade pode estar mais fortemente relacionada à ansiedade perinatal do que DPP (PAUL IM, et al., 2013). No entanto, em contraste, não encontrou nenhum significado e não consigo estabelecer uma relação entre primiparidade e ansiedade durante a gravidez (HENDERSON J e REDSHAW M, 2013). Ainda, alguns estudos descobriram que a DPP está relacionada a interrupção precoce da amamentação, ou a não amamentação (CLOUT D, et al., 2015).

Estudos recentes descobriram que mães com medo intenso do parto eram mais propensas a ter cesáreas eletivas e frequentemente experimentam sintomas elevados de depressão e ansiedade. Contudo, pouco se sabe sobre as mudanças no humor deprimido e na ansiedade ao longo do tempo entre mulheres que se submetem a uma cesárea eletiva. Até o momento, poucos estudos acompanharam mulheres submetidas a uma cesárea eletiva desde a gravidez até o parto (KUO S, et al., 2014). Ademais, estresse da paternidade, falta de apoio do parceiro ou até a sua ausência, dor corporal, baixa escolaridade, preocupações financeiras, tabagismo são fatores predisponentes para DPP (QUAN C, et al., 2019; ZACONETA AM, et al., 2013; SADAT Z, et al., 2014).

Uma metanálise de estudos de caso-controle e de coorte estudou a associação entre o parto cesáreo e DPP. aponta para vários mecanismos que explicam a relação entre a cesariana e DPP: as alterações biológicas que ocorrem no corpo da mãe; cesariana sendo um fator de risco para hemorragia, o que aumenta a probabilidade de DPP; cesariana aumentando o risco de mortalidade infantil; falha na amamentação; mulheres com tendência à depressão, estando potencialmente geralmente ansiosas para o parto (MOAMERI $\mathrm{H}$, et al., 2019).

A cesariana provoca alterações biológicas no corpo da mãe, incluindo uma diminuição no nível de prolactina e um aumento no nível de interleucina-6. Essas mudanças cortam os fatores de risco para DPP. Ademais, a cesariana é um fator de risco para hemorragia e mortalidade infantil e, como consequência, aumenta o risco de DPP. No mesmo estudo, aproximadamente metade das mulheres avaliou suas experiências de parto como moderadas, ruins e muito ruins, e havia risco de DPP em aproximadamente um terço delas. O risco de DPP das mães que tiveram parto vaginal e que receberam uma intervenção (enema, indução de ocitocina e amniotomia) durante o parto foi considerado alto. Ainda foi evidenciada uma correlação negativa entre o uso comum de intervenções como monitoramento fetal eletrônico, episiotomia, laceração perineal, indução, enema e a satisfação da mãe pelo nascimento. Além disso, as cesarianas de urgência podem ter um efeito negativo na situação emocional. A dor do parto e as intervenções de rotina durante o parto contribuem para um aumento da ansiedade e decepção na gestante e é apontada como uma das causas importantes de DPP (MOAMERI H, et al., 2019).

O parto cesáreo foi associado a altos níveis de depressão, ansiedade e estresse pós-parto. Problemas de sono infantil foram relacionados à depressão materna, problemas de saúde infantil foram relacionados à ansiedade materna, alto estresse e problemas de sono materno foram relacionados ao DPP. No entanto, os resultados se tornaram não significativos após o controle dos níveis de sofrimento pré-natal. Por fim, as mulheres que realizaram parto cesáreo apresentaram níveis mais elevados de estresse, ansiedade e depressão pré-natal em relação às mulheres que não realizaram o procedimento (MOAMERI H, et al., 2019).

Uma meta-análise revelou que a avaliação da dor do parto e da dor no puerpério inicial diferiu de acordo com o tipo de parto vivenciado pela mulher. $O$ estudo mostrou que as mulheres classificaram a intensidade da dor como a mais alta durante o parto vaginal e cesárea de emergência, e a mais baixa durante a cesariana eletiva. No entanto, no momento da alta hospitalar, as mulheres após o parto vaginal sentiram menos dor, enquanto a maioria das dores foi sentida pelas mulheres após a cesariana (tanto de emergência quanto eletiva) (MOAMERI H, et al., 2019). Além disso, a pesquisas demonstraram que a dor após a cirurgia de cesariana persiste por até 6 meses após o parto em $33 \%$ das mulheres, em comparação com $2 \%$ das mulheres após o parto vaginal (ILSKA M, et al., 2020). 


\section{Impacto familiar da Depressão Pós-Parto}

A DPP afeta negativamente as mães, na medida em que compromete a interação materno-infantil, além de representar um fator de risco para a interrupção precoce da amamentação e de trazer consequências para a criança (HANLON AJM e BECKMANN MM, 2015). Concernente ao quadro, a DPP está relacionada ao aumento da incidência de infanticídio, de automutilação e de ideação suicida maternas. As consequências mais comuns desse transtorno para as crianças incluem dificuldades emocionais e cognitivas, problemas comportamentais, baixa competência social, atraso cognitivo e prejuízo nas interações sociais das crianças (XU H, et al., 2017; HANLON AJM e BECKMANN MM, 2015). Em adição a influenciar na saúde materna e no crescimento da criança, a DPP atua no desenvolvimento e na depressão paterna. Ademais, a função sexual feminina após o parto pode ser afetada da mesma forma pela DPP (CHANG SR, et al., 2014).

A ansiedade materna persistente e a gravidade dos sintomas depressivos durante os primeiros dois anos após o parto estão relacionados a relatos maternos de pior comportamento e negatividade emocional em seus filhos aos 24 meses após o parto, destacando o potencial impacto negativo da depressão e ansiedade materna persistente nos resultados emocionais e comportamentais das crianças (PRENOVEAU JM, et al., 2017). Os sintomas depressivos maternos nas primeiras 10 semanas após o parto predizem o atraso no desenvolvimento das habilidades de comunicação não-verbal de bebês aos 14 meses de idade. Além disso, os sintomas depressivos maternos após o parto foram relatados como preditivos de atraso de linguagem dos bebês, influenciando o desenvolvimento de habilidades sociais iniciais (KAWAI E, et al., 2017), da mesma forma que o comprometimento da saúde mental das mães no período pós-parto também aumenta os gastos gerais com saúde (CHEN HH, et al., 2017).

Sendo assim, dado o enorme impacto da DPP sobre os indivíduos, suas famílias e suas condições econômicas, a identificação de fatores de risco para a morbidade psicológica pós-parto pode permitir a intervenção de profissionais de saúde para prevenir e detectar os sintomas precocemente e tratar e apoiar as mulheres com DPP (HANLON AJM e BECKMANN MM, 2015). Destaca-se a importância da intervenção precoce e, se necessário, sustentada para mães que estão em risco de depressão persistente nos primeiros anos de vida de seus filhos. Intervenções psicológicas e psicossociais (como terapia cognitivocomportamental, psicoterapia psicodinâmica ou aconselhamento), tratamentos farmacológicos ou terapia hormonal podem ser fornecidos para tratar transtornos mentais pós-parto, como os sintomas de estresse pósparto, ansiedade e depressão (PRENOVEAU JM, et al., 2017), visto que foram relatadas associações significativas entre esses três diagnósticos e o parto cesáreo e as suas consequências (CHEN HH, et al., 2017).

\section{CONSIDERAÇÕES FINAIS}

Observa-se a partir da literatura consultada que a DPP consiste num adoecimento que afeta a saúde da mulher, com repercussões na interação social da mãe com o seu bebê e com toda família. Os estudos revisados indicam que a depressão pós parto tem fatores de risco multifatoriais, podendo destacar o histórico de depressão na gestação, estresse, ansiedade, entre outros. Portanto, é indispensável a avaliação precoce da DPP pelas equipes multidisciplinares, visto que muitos dos fatores de risco identificados são considerados modificáveis. Faz-se necessário ressaltar e alertar os profissionais de saúde sobre a importância do papel que desempenham na detecção dos sinais e sintomas da DPP precocemente, a fim de evitar a DPP e as possíveis repercussões na interação mãe-filho.

\section{REFERÊNCIAS}

1. ATAN ŞU, et al. Relation between mothers' types of labor, birth interventions, birth experiences and postpartum depression: A multicentre follow-up study. Sexual \& Reproductive Healthcare, 2018; 18: 13-18.

2. BELL AF, ANDERSON E. The birth experience and women's postnatal depression: A systematic review. Midwifery. 2016; 39: 112-123.

3. CHANG SR, et al. Depressive symptoms, pain, and sexual dysfunction over the first year following vaginal or cesarean delivery: A prospective longitudinal study. International Journal of Nursing Studies, 2014; 52(9): 1433-1444. 
4. $\mathrm{CHEN} \mathrm{HH}$, et al. Understanding the relationship between cesarean birth and stress, anxiety, and depression after childbirth: A nationwide cohort study. Birth. 2017; 44(4): 369-376.

5. CIRIK DA, et al. The impact of prenatal psychologic and obstetric parameters on postpartum depression in late-term pregnancies: A preliminary study. Taiwanese Journal of Obstetrics \& Gynecology, 2016; 55: 374-378.

6. CLOUT D, BROWN RF. Sociodemographic, pregnancy, obstetric, and postnatal predictors of postpartum stress, anxiety and depression in new mothers. Journal of Affective Disorders, 2015; 188: 60-67.

7. FARR SL, et al. Postpartum Anxiety and Comorbid Depression in a Population - Based Sample of Women. J Womens Health (Larchmt). 2014; 23(2): 120-128.

8. HANLON AJM, BECKMANN MM. Mode of birth and early postnatal psychological morbidity. Australian and New Zealand Journal of Obstetrics and Gynaecology, 2015; 55(6): 578-583.

9. HENDERSON J, REDSHAW M. Anxiety in the perinatal period: antenatal and postnatal influences and women's experience of care. J. Reprod. Infant Psychol, 2013; 31(5): 465-478.

10. ILSKA M, et al. Vaginal delivery or caesarean section - Severity of early symptoms of postpartum depression and assessment of pain in Polish women in the early puerperium. Midwifery, 2020; 87: 1-6.

11. KAWAI E, et al. Maternal postpartum depressive symptoms predict delay in non-verbal communication in 14-monthold infants. Infant Behavior and Development, 2017; 46, 33-45.

12. KUO SY, et al. Depression and Anxiety Trajectories among Women Who Undergo an Elective Cesarean Section. PLoS One, 2017; 9(1): 1-8.

13. MAHISHADE AV, BHATT JA. Comparison of level of depression among mothers with lower segment cesarean section and vaginal delivery: A cross-sectional study. Journal of the Scientific Society, 2017; 44(1): 15-19.

14. MOAMERI $\mathrm{H}$, et al. Association of postpartum depression and cesarean section: A systematic review and metaanalysis. Clinical Epidemiology and Global Health, 2019; 7(3): 471-480

15. OLIEMAN RM, et al. The effect of an elective cesarean section on maternal request on peripartum anxiety and depression in women with childbirth fear: a systematic review. BMC Pregnancy and Childbirth, 2017; 17(1): $195-203$.

16. PAUL IM, et al. Postpartum anxiety and maternal-infant health outcomes. Pediatrics, 2013; 131(4): 1218-1224.

17. PRENOVEAU JM, et al. Supplemental Material for Maternal Postnatal Depression and Anxiety and Their Association With Child Emotional Negativity and Behavior Problems at Two Years. Developmental Psychology, 2017; 53(1): 5062.

18. QUAN C, et al. The role of kynurenine pathway and kynurenic aminotransferase alleles in postpartum depression following cesarean section in Chinese women. Wiley Periodicals, 2019; 10(4): 1-9.

19. SADAT Z, et al. Effect of mode of delivery on postpartum depression in Iranian women. Journal of Obstetrics and Gynaecology Research, 2014; 40(1): 172-177.

20. TATA N, et al. Predictors of Fear of Childbirth in the Primiparous Women in Ardabil-Iran. Journal of Clinical and Diagnostic Research, 2019, 13(7): 1-5.

21. WALLWIENER S, et al. Epidemiology of mental disorders during pregnancy and link to birth outcome: a large-scale retrospective observational database study including 38,000 pregnancies. Arch Gynecol Obstet, 2019; 299(3): 755763.

22. $\mathrm{XU} \mathrm{H}$, et al. Cesarean section and risk of postpartum depression: A meta-analysis. Journal of Psychosomatic Research, 2017; 97: 118-126.

23. ZACONETA AM, et al. Depression with postpartum onset: a prospective cohort study in women undergoing elective cesarean section in Brasilia, Brazil. Revista Brasileira Ginecologia Obstetrícia. 2013, 35(3): 130-5.

24. ZANARDO V, et al. The role of elective and emergency cesarean delivery in maternal postpartum anhedonia, anxiety, and depression. Int J Gynecol Obstet, 2017; 143(3): 1-16. 